FluoroSpot) and cytotoxic activity (Delfia assay) have been assessed upon the co-culture with CD19+ or CD19- target cells.

Results Enrichment of CD4+CAR $+\mathrm{T}$ cells, besides CD8 $+\mathrm{CAR}+$, were observed in UCB-CAR- vs. PBL-CAR-T cells (40-59\% of positive cells; as well as of CD45RA+ cells (4060 vs. $20-30 \%$ of positive cells; $p<0.05)$. The preferential selection of early stage of differentiation (CCR7+CD28 $+\mathrm{CD} 27+\mathrm{CD} 137+\mathrm{CD} 62 \mathrm{~L}+$ ) for CAR-T cells isolated from both source of lymphocytes occurred. LAG3 and TIM-3 expressing $\mathrm{T}$ cells were found with higher frequency in UCBvs. PBL-CAR-T cells, with superior association with $\mathrm{CD} 4+$ UCB-derived cells. CD19-CAR-T cells secreted IFN-g(300-400 $\mathrm{N}$. spot/10 $\times 104 \mathrm{~T}$ cells), regardless the co-stimulatory molecules (CD28z vs $4-1 \mathrm{BBz}$ ), upon the engagement of CAR by CD19. A minority of IL-4 releasing T cells was found for few CAR-T cells activated with TransAct. IFN-gamma secreting CAR-T cells simultaneously released IL-2, Granzyme B and Perforin but not IL-5 and IL-17, thus belonging to TH-1/effector subset. The cytotoxic activity of these $\mathrm{T}$ cells against CD19+ target cells was also determined by europium release assay. Differential gene expression profile was determined in UCB-CAR-T vs. PBL-CAR-T cells bearing the different CARs following the co-culture with either CD19+ or CD19- target cells.

Conclusions The deep characterization of CD19-CAR-T cells contributed to validate the generation of anti-tumor 'off-theshelf' CAR-T cells from UCB.

Ethics Approval The study was approved by Sidra Medicine's Ethics Board, approval number 1812044429.

http://dx.doi.org/10.1136/jitc-2020-SITC2020.0124

\section{REEXAMINATION OF MAGE-A3 AS A T-CELL THERAPEUTIC TARGET}

Aaron Martin*, Xueyin Wang, Han Xu, Alexander Kamb, Mark Sandberg, Kathleen Negri, Ming Wu, Dora Toledo Warshaviak, Grant Gabrelow, Michele Mcelvain, Mark Daris, Bella Lee. A2 Biotherapeutics, Thousand Oaks, CA, USA

Background Recurrent cancer-specific targets are rare. Given the pace of genomic research over the past three decades, few are likely to lie yet undiscovered. In 2013 an innovative MAGE-A3-directed cancer therapeutic of great potential value was terminated in the clinic because of neurotoxicity. ${ }^{1}$ The safety problems were hypothesized to originate from off-target TCR activity against a closely related MAGE-A12 peptide.

Methods A combination of published and new data led us to test this hypothesis with current technology, including RNA hybridization in situ and further analysis of the clinical TCR's specificity to MAGE-A12 and other antigens.

Results We find that a key prediction of the MAGE-A12 toxicity hypothesis, the existence of rare, high-MAGE-A12expressing cells in the brain, is not supported by the data. Our results imply that an alternative related peptide from the EPS8L2 protein is more likely responsible for the toxicity. Therefore, it may be valuable to reconsider MAGE-A3 as a cancer target using HLA-A*02-restricted-TCRs or CARs. As a step in this direction, we isolated MAGE-A3 pMHC-directed CARs, targeting the same peptide as the clinical TCR. These CARs have high selectivity, and avoid cross-reaction with the EPS8L2 peptide that represents a significant risk for MAGEA3-targeted therapeutics.
Conclusions Given the qualities of MAGE-A3 as an onco-testis antigen widely expressed in tumors and largely absent from normal adult tissues, our findings suggest that MAGE-A3 may deserve further consideration as a cancer target. We have identified CARs with selectivity profiles consistent with a cell therapeutic directed against HLA-A*02-positive, MAGE-A3expressing cancers. The relative merits of TCRs and CARs for this target will be discussed.

\section{REFERENCE}

1. Morgan RA, Chinnasamy N, Abate-Daga D, Gros A, Robbins PF, Zheng Z, Dudley ME, Feldman SA, Yang JC, Sherry RM, et al. Cancer regression and neurological toxicity following anti-MAGE-A3 TCR gene therapy. J Immunother 2013;36:133151, doi:10.1097/CJI.0b013e3182829903.

http://dx.doi.org/10.1136/jitc-2020-SITC2020.0125

\section{EARLY-PHENOTYPE LEWIS Y CAR-T CELLS PERSIST BETTER IN VIVO AND INDUCE SOLID TUMOR REGRESSION IN COMBINATION WITH ANTI-PD1}

'Deborah Meyran*, 'Joe Zhu, 'Jeanne Butler, 'Sean Macdonald, 'Daniela Tantalo, ${ }^{1}$ Niko Thio, ${ }^{1}$ Kevin Sek, ${ }^{2}$ Paul Ekert, ${ }^{1}$ Michael Kershaw, ${ }^{1} J o e$ Trapani, ${ }^{1}$ Phillip Darcy, ${ }^{1}$ Paul Neeson. ${ }^{1}$ Peter MacCallum Cancer Centre, Melbourne, Australia; ${ }^{2}$ Children's Cancer Institute, Sydney, Australia

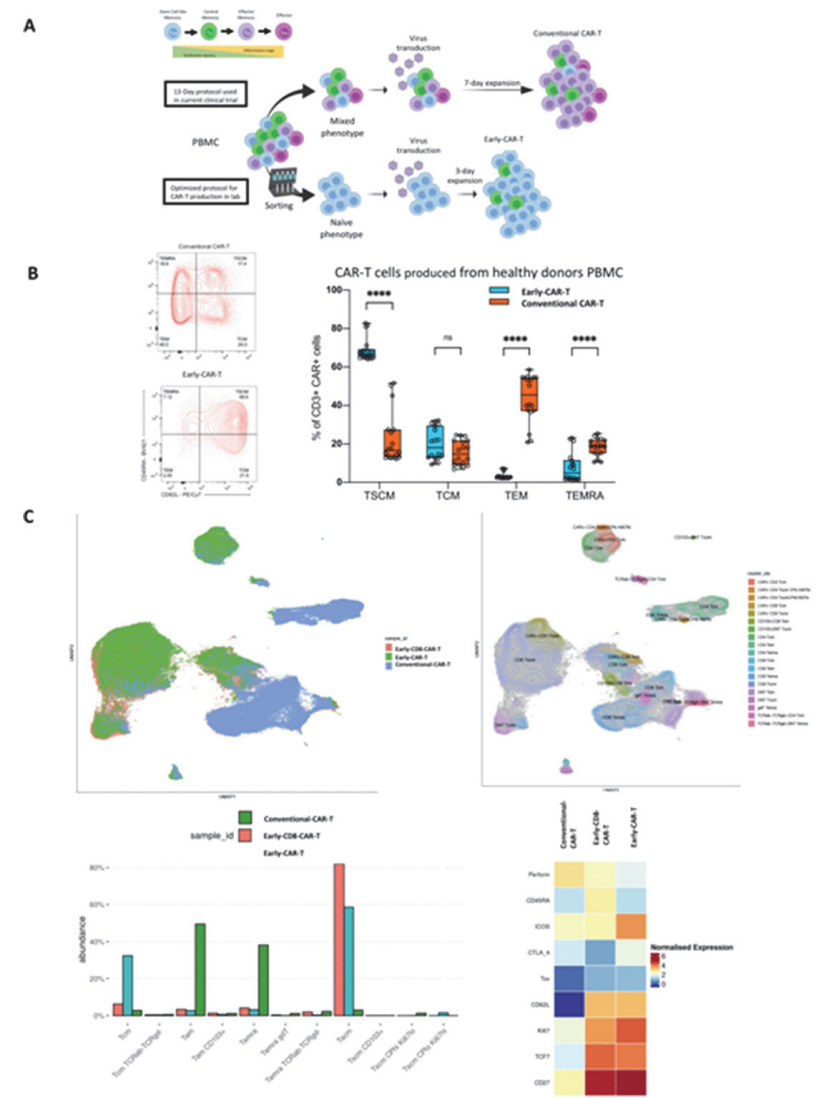

Abstract 126 Figure 1 Early-CAR-T protocol, including Naïve-T cells purification and expansion in IL-7 and IL-15 promotes the maintenance of a TSCM and TCM phenotype. A) Scheme of the 7-day production protocol for Early-CAR-T cells. B) Phenotype by FACs of the conventional CAR-T cells and the Early-CAR-T cells. Pooled data in triplicate for 6 donors. C) Phenotype by Mass cytometry comparing the Conventional-CAR-T cells vs Early-CAR-T cells vs Early-CD8-CAR-T cells. Data for one donor representative of 3 different donors 